\title{
GROWTH OPTIMAL INVESTMENT WITH THRESHOLD REBALANCING PORTFOLIOS UNDER TRANSACTION COSTS
}

\author{
Sait Tunc ${ }^{1}$, Mehmet A. Donmez ${ }^{2}$ and Suleyman S. Kozat ${ }^{3}$ \\ ${ }^{1}$ Georgia Institute of Technology, Industrial Engineering Department, Atlanta, USA \\ ${ }^{2}$ Koc University, Electrical and Electronics Engineering Department, Istanbul, Turkey \\ ${ }^{3}$ Bilkent University, Electrical and Electronics Engineering Department, Ankara, Turkey
}

\begin{abstract}
We study how to invest optimally in a stock market having a finite number of assets from a signal processing perspective. In particular, we introduce a portfolio selection algorithm that maximizes the expected cumulative wealth in i.i.d. twoasset discrete-time markets where the market levies proportional transaction costs in buying and selling stocks. This is achieved by using "threshold rebalanced portfolios", where trading occurs only if the portfolio breaches certain thresholds. Under the assumption that the relative price sequences have log-normal distribution from the Black-Scholes model, we evaluate the expected wealth under proportional transaction costs and find the threshold rebalanced portfolio that achieves the maximal expected cumulative wealth over any investment period.
\end{abstract}

Index Terms - Portfolio management, threshold rebalancing, transaction cost, discrete-time market, continuous distribution.

\section{INTRODUCTION}

Recently financial applications attracted a significant interest from the signal processing community since the recent global crises demonstrated the importance of sound financial modeling and reliable data processing [1,2]. Stock markets produce vast amount of temporal data ranging from stock prices to interest rates making them ideal mediums to apply signal processing methods. Furthermore, due to the integration of high performance, low-latency computing recourses and financial data collection infrastructures, a wide range of signal processing algorithms could be readily leveraged with full potential in stock markets. This paper specifically focuses on the portfolio selection problem, which is one the most important financial applications and has already attracted substantial interest from the signal processing community [3-8].

Determination of the optimum portfolio and the best portfolio rebalancing strategy that maximize wealth in discretetime markets with no transaction fees is heavily investigated in information theory $[9,10]$, machine learning [11-13] and signal processing [14-17] fields. Assuming that the portfolio rebalancings, i.e., adjustments to the portfolio by buying and selling stocks, require no transaction fees and with some further mild assumptions on the stock prices, the portfolio that achieves the maximum expected wealth is shown to be a constant rebalanced portfolio (CRP) $[10,18]$. A CRP is a portfolio strategy where the distribution of funds over the stocks are reallocated to a predetermined structure, also known as the target portfolio, at the start of each investment period. However, we emphasize that maintaining a CRP requires potentially significant trading due to possible rebalancings at each investment period [14]. As shown in [14], even the performance of the best CRP is severely affected by moderate transaction fees rendering CRPs ineffective in real life stock markets. Clearly, one can potentially obtain significant gain in wealth by including unavoidable transactions fees in the market model and perform reallocation accordingly.

In these lines, the optimal portfolio selection problem under transactions costs is extensively investigated for continuous-time markets [19-22], where growth optimal policies that keep the portfolio in closed compact sets by trading only when the portfolio hits the compact set-boundaries are introduced. It has been shown in [23] that under certain mild assumptions on the sequence of stock prices, similar no trade zone portfolios achieve the optimal growth rate even for discrete-time markets under proportional transaction costs. For markets having two stocks, i.e., two-asset stock markets, these no trade zone portfolios correspond to threshold portfolios, i.e., the no trade zone is defined by thresholds around the target portfolio. In particular, unlike a calendar rebalancing portfolio, e.g., a CRP, a threshold rebalanced portfolio (TRP) rebalances by buying and selling stocks only when the portfolio breaches the preset boundaries, or "thresholds", and otherwise does not perform any rebalancing. Intuitively, by limiting the number of rebalancings due to these non rebalancing regions, threshold portfolios are able to avoid hefty transactions costs associated with excessive trading unlike calendar portfolios. Although TRPs are shown to be optimal in i.i.d. discrete-time two-asset markets (under certain technical conditions) [23], finding the TRP that maximizes the expected growth of wealth under proportional transaction costs is not solved, except for basic scenarios [23], to the best of our knowledge.

In this paper, we first evaluate the expected wealth achieved by a TRP over any finite investment period given any target portfolio and threshold for two-asset discrete-time stock markets subject to proportional transaction fees. We emphasize that we study the two-asset market for notational simplicity and our derivations can be readily extended to markets having more than two assets as provided in the paper where needed. We consider i.i.d. discrete-time markets represented by the sequence of price relatives (defined as the ratio of the closing prices of stocks in consecutive days), where 
the sequence of price relatives follow log-normal distributions. Note that the log-normal distribution is the assumed statistical model for price relative vectors in the well-known Black-Scholes model [24, 25] and this distribution is shown to accurately model real life stock prices by many empirical studies [24]. Under this setup, we provide an iterative relation that efficiently and recursively calculates the expected growth over any period in any i.i.d. discrete-time market. This expected growth is then optimized by a brute force method to yield the optimal target portfolio and threshold to maximize the expected wealth over any investment period. We also illustrate the performance of our algorithm under different scenarios demonstrating its effectiveness.

We begin with the detailed description of the market and the TRPs in Section 2. We then calculate the expected wealth using a TRP in an i.i.d. two-asset discrete-time market under proportional transaction costs over any investment period in Section 3. We provide an iterative relation to recursively calculate the expected wealth growth. The paper is then concluded with the simulations of given algorithm in Section 4 .

\section{PROBLEM DESCRIPTION}

In this paper, all vectors are column vectors and represented by lower-case bold letters. Consider a market with $m$ stocks and let $\{\mathbf{x}(t)\}_{t \geq 1}$ represent the sequence of price relative vectors in this market, where $\mathrm{x}(t)=\left[x_{1}(t), x_{2}(t), \ldots, x_{m}(t)\right]^{T}$ with $x_{i}(t) \in \mathbb{R}^{+}$for $i \in\{1,2, \ldots, m\}$ such that $x_{i}(t)$ represents the ratio of the closing price of the $i$ th stock for the $t$ th trading period to that from the $(t-1)$ th trading period. At each investment period, say period $t, \mathbf{b}(t)$ represents the vector of portfolios such that $b_{i}(t)$ is the fraction of money invested on the $i$ th stock. We allow only long-trading such that $\sum_{i=1}^{m} b_{i}(t)=1$ and $b_{i}(t) \geq 0$. After the price relative vector $\mathbf{x}(t)$ is revealed, we earn $\mathbf{b}^{T}(t) \mathbf{x}(t)$ at the period $t$.

We denote a TRP with a target vector $\mathbf{b}$ and a threshold $\epsilon$ (with certain abuse of notation) as "TRP with $(\mathbf{b}, \epsilon)$ ". For a sequence of price relatives vectors $\mathrm{x}^{n} \triangleq[\mathrm{x}(1), \mathrm{x}(2), \ldots, \mathrm{x}(n)]$ with $\mathrm{x} \in \mathbb{R}_{m}^{+}$, a TRP with $(\mathbf{b}, \epsilon)$ rebalances the portfolio to $\mathbf{b}$ at the first time $\tau$ satisfying

$$
\frac{b_{j} \prod_{t=1}^{\tau} x_{j}(t)}{\sum_{k=1}^{m} b_{k} \prod_{t=1}^{\tau} x_{k}(t)} \notin\left[b_{j}-\epsilon_{j}, b_{j}+\epsilon_{j}\right]
$$

for any $j \in\{1,2, \ldots, m\}$, thresholds $\epsilon_{j}$, and does not rebalance otherwise, i.e., while the portfolio vector stays in the no rebalancing region. Starting from the first period of a no rebalancing region, i.e., where the portfolio is rebalanced to the target portfolio $\mathbf{b}$, say $t=1$ for this example, the wealth gained during any no rebalancing region is given by

$$
S\left(\mathbf{x}^{n} \mid \mathbf{b}^{n} \in \mathcal{E}_{n}^{\mathrm{nc}}\right)=\sum_{k=1}^{m} b_{k} \prod_{t=1}^{n} x_{k}(t),
$$

where $\mathbf{b}^{n}=[\mathbf{b}(1), \mathbf{b}(2), \ldots, \mathbf{b}(n)], \mathbf{b}(t)$ is the portfolio at the period $t$ and $\mathcal{E}_{n}^{\text {nc }}$ is the length $n$ no rebalancing region defined as

$$
\begin{gathered}
\mathcal{E}_{n}^{\mathrm{nc}}=\left\{\mathbf{b}^{n} \mid \mathbf{b}(1)=\mathbf{b}, b_{j}(t) \in\left(b_{j}-\epsilon_{j}, b_{j}+\epsilon_{j}\right),\right. \\
j \in\{1,2, \ldots, m\}, t \in\{1,2, \ldots, n\}\} .
\end{gathered}
$$

A TRP pays a transaction fee when the portfolio vector leaves the predefined no rebalancing region, i.e., goes out of the no rebalancing region $\mathcal{E}_{n}^{\text {nc }}$, and rebalanced back to its target portfolio vector $b$. Since the TRP may avoid constant rebalancing, it may avoid excessive transaction fees while securing the portfolio to stay close to the target portfolio $b$, when we have heavy transaction costs in the market.

For notational clarity, in the remaining of the paper, we assume that the number of stocks in the market is equal to 2, i.e., $m=2$. Note that our results can be readily extended to the case when $m>2$. Then, the threshold rebalanced portfolios are described as follows.

Given a TRP with target portfolio $\mathbf{b}=[b, 1-b]^{T}$ with $b \in[0,1]$ and a threshold $\epsilon$, the no rebalancing region of a TRP with $(\mathbf{b}, \epsilon)$ is represented by $(b-\epsilon, b+\epsilon)$. Given a TRP with $(b-\epsilon, b+\epsilon)$, we only rebalance if the portfolio leaves this region, which can be found using only the first entry of the portfolio (since there are two stocks), i.e., if $b_{1, \text { old }}(t) \notin$ $(b-\epsilon, b+\epsilon)$. In this case, we rebalance $b_{1, \text { old }}(t)$ to $b$.

In this paper, we assume that the price relative vectors have a log-normal distribution following the well-known Black-Scholes model [24]. This distribution, which is extensively used in the financial literature, is shown to model empirical price relative vectors close to accurate in many tests [26]. Hence, we assume that $\mathbf{x}(t)=\left[x_{1}(t), x_{2}(t)\right]^{T}$ has an i.i.d. log-normal distribution with mean $\boldsymbol{\mu}=\left[\mu_{1}, \mu_{2}\right]$ and standard deviation $\boldsymbol{\sigma}=\left[\sigma_{1}, \sigma_{2}\right]$, respectively, i.e., $\mathbf{x}(t) \sim \ln \mathcal{N}\left(\boldsymbol{\mu}, \boldsymbol{\sigma}^{2}\right)$.

\section{THRESHOLD REBALANCED PORTFOLIOS}

In this section, we analyze the TRPs in a discrete-time market with proportional transaction costs as defined in Section 2. We first introduce an iterative relation, as a theorem, to recursively evaluate the expected achieved wealth of a TRP over any investment period. The terms in this iterative equation are calculated using a certain form of multivariate Gaussian integrals. We then use the given iterative equation to find the optimal $\epsilon$ and $b$ that maximize the expected wealth over any investment period.

\subsection{An Iterative Relation to Calculate the Expected Wealth}

In this section, we introduce an iterative equation to evaluate the expected cumulative wealth of a TRP with $(b-\epsilon, b+\epsilon)$ over any period $n$, i.e., $E[S(n)]$. For a TRP with $(b-\epsilon, b+\epsilon)$, any investment scenario can be decomposed as the union of consecutive no-crossing blocks such that each rebalancing instant, to the initial b, signifies the end of a block. Hence, based on this observation, the expected gain of a TRP between consecutive crossings, i.e. the gain during the no-rebalancing regions, is directly proportional to the overall wealth growth. Therefore, in the next we first calculate the conditional expected gain of a TRP over no rebalancing regions and then introduce the iterative relation based on these derivations.

For a TRP with $(b-\epsilon, b+\epsilon)$, we call a no rebalancing region of length $n$ as "period $n$ with no-crossing" such that the TRP with the initial and target portfolio $\mathbf{b}=[b, 1-b]$ stays in the $(b-\epsilon, b+\epsilon)$ interval for $n-1$ consecutive investment periods and crosses one of the thresholds at the $n$th period. 
We next calculate the expected gain of a TRP over any nocrossing period as follows.

The wealth growth of a TRP with $(b-\epsilon, b+\epsilon)$ for a period $\tau$ with no-crossing can be written as [27]

$$
S(\tau)=\zeta_{1} \prod_{t=1}^{\tau}\left[x_{1}(t)\right]+\zeta_{2} \prod_{t=1}^{\tau}\left[x_{2}(t)\right]
$$

where $\zeta_{1} \triangleq b-2 c\left(b-b^{2}\right), \zeta_{2} \triangleq 1-b+2 c\left(b-b^{2}\right)$ for $b+\epsilon$ hitting and $\zeta_{1} \triangleq b+2 c\left(b-b^{2}\right), \zeta_{2} \triangleq 1-b-2 c\left(b-b^{2}\right)$ for $b-\epsilon$ hitting and $c$ represents the symmetrical commission cost, to rebalance two stocks, i.e., $b_{1 \text {,old }}(\tau+1)$ to $b$, and $b_{2, \text { old }}(\tau+$ $1)=1-b_{1, \text { old }}(\tau+1)$ to $1-b$. Thus, the conditional expected gain of a TRP conditioned on that the portfolio stays in a no rebalancing region until the last period of the region can be found by calculating the expected value of (4).

In order to calculate the expected wealth $E[S(n)]$ iteratively, let us first define the variable $R(\tau)$, which is the expected cumulative gain of all possible portfolios that hit any of the thresholds first time at the $\tau$ th period, i.e.,

$$
R(\tau)=E\left[S(\tau) \mid \mathbf{b}^{\tau} \in \mathcal{E}_{\tau}^{\mathrm{fc}}\right]
$$

where $\mathcal{E}_{\tau}^{\mathrm{fc}}$ denotes the set of all possible portfolios with initial portfolio $b$ and that stay in the no rebalancing region for $\tau-1$ consecutive periods and hits one of the $b-\epsilon$ or $b+\epsilon$ boundary at the $\tau$ th period, i.e.,

$$
\begin{gathered}
\mathcal{E}_{\tau}^{\mathrm{fc}_{\mathrm{c}} \triangleq}\left\{\mathbf{b}^{\tau} \in \mathcal{B}_{\tau}(b, \epsilon) \mid b(1)=b, b(i) \in(b-\epsilon, b+\epsilon)\right. \\
\forall i \in\{2, \ldots, \tau-1\}, b(\tau) \notin(b-\epsilon, b+\epsilon)\} .
\end{gathered}
$$

Here, $\mathcal{B}_{\tau}(b, \epsilon)$ is defined as the set of all possible threshold rebalanced portfolios with initial and target portfolio $b$ and a no rebalancing interval $(b-\epsilon, b+\epsilon)$. Similarly we define the variable $T(\tau)$, which is the expected growth of all possible portfolios of length $\tau$ with no threshold crossings, i.e.,

$$
T(\tau)=E\left[S(\tau) \mid \mathbf{b}^{\tau} \in \mathcal{E}_{\tau}^{\mathrm{nc}}\right],
$$

where $\mathcal{E}_{\tau}^{\text {nc }}$ denotes the set of portfolios with initial portfolio $b$ and that stay in the no rebalancing region for $\tau$ consecutive periods, i.e., ${ }^{1}$

$$
\begin{aligned}
\mathcal{E}_{\tau}^{\mathrm{nc}} \triangleq & \left\{\mathbf{b}^{\tau} \in \mathcal{B}_{\tau}(b, \epsilon) \mid b(1)=b, b(i) \in[b-\epsilon, b+\epsilon]\right. \\
& \forall i \in\{2, \ldots, \tau\}\} .
\end{aligned}
$$

Given the variables $R(\tau)$ and $T(\tau)$, we next introduce a theorem that iteratively calculates the expected wealth growth of a TRP over any period $n$. Hence, to calculate the expected achieved wealth, it is sufficient to calculate $R(\tau), T(\tau)$, threshold crossing probabilities $P\left(\mathbf{b}^{n} \in \mathcal{E}_{n}^{\mathrm{fc}}\right)$ and $P\left(\mathbf{b}^{n} \in \mathcal{E}_{n}^{\text {nc }}\right)$, which are explicitly evaluated in the next section.

${ }^{1}$ This is the special case of the definition in (3) for $m=2$.
Theorem 3.1 The expected wealth growth of a TRP $(b-\epsilon, b+$ $\epsilon)$, i.e., $E[S(n)]$, over any i.i.d. sequence of price relative vectors $\mathrm{x}^{n}=[\mathrm{x}(1), \mathrm{x}(2), \ldots, \mathrm{x}(n)]$, satisfies

$$
E[S(n)]=\sum_{i=1}^{n} P\left(\mathcal{E}_{i}^{\mathrm{fc}}\right) R(i) E[S(n-i)]+P\left(\mathcal{E}_{n}^{\mathrm{nc}}\right) T(n),
$$

where we define $S_{0}=1, R(n)$ in (5), $T(n)$ in (7), $\mathcal{E}_{i}^{\mathrm{fc}}$ in (6) and $\mathcal{E}_{n}^{\mathrm{nc}}$ in (8).

The proof of the Theorem 3.1 can be found in [27]. Theorem 3.1 provides a recursion to iteratively calculate the expected wealth growth $E[S(n)]$, when $R(\tau)$ and $T(\tau)$ are explicitly calculated for a TRP with $(b-\epsilon, b+\epsilon)$. Hence, if we can obtain $P\left(\mathcal{E}_{\tau}^{\mathrm{fc}}\right) R(\tau)$ and $P\left(\mathcal{E}_{\tau}^{\mathrm{nc}}\right) T(\tau)$ for any $\tau$, then (9) yields a simple iteration that provides the expected wealth growth for any period $n$. We next give the explicit definitions of the events $\mathbf{b}^{\tau} \in \mathcal{E}_{\tau}^{\text {fc }}$ and $\mathbf{b}^{\tau} \in \mathcal{E}_{\tau}^{\text {nc }}$ in order to calculate the conditional expectations $R(\tau)$ and $T(\tau)$. Following these definitions, we calculate $P\left(\mathcal{E}_{\tau}^{\mathrm{fc}}\right) R(\tau)$ and $P\left(\mathcal{E}_{\tau}^{\text {nc }}\right) T(\tau)$ to evaluate the expected wealth growth $E[S(\tau)]$, iteratively from Theorem 3.1 and find the optimal TRP, i.e., optimal $b$ and $\epsilon$, by using a brute force search.

We next provide the conditions for the market portfolios to cross the corresponding thresholds and calculate the probabilities for the events $\mathbf{b}^{\tau} \in \mathcal{E}_{\tau}^{\text {fc }}$ and $\mathbf{b}^{\tau} \in \mathcal{E}_{\tau}^{\text {nc }}$. We then calculate the conditional expectations $R(n)$ and $T(n)$ as certain multivariate Gaussian integrals.

Hence, we can explicitly describe the event that the market threshold portfolio $(b-\epsilon, b+\epsilon)$ does not hit any of the thresholds for $\tau$ consecutive periods, $\mathbf{b}^{\tau} \in \mathcal{E}_{\tau}^{\text {nc }}$, as the intersection of the events as [27]

$$
\mathbf{b}^{\tau} \in \mathcal{E}_{\tau}^{\mathrm{nc}} \equiv \bigcap_{i=1}^{\tau}\left\{\gamma_{2} \Pi_{1}(i) \leq \Pi_{2}(i) \leq \gamma_{1} \Pi_{1}(i)\right\}
$$

where $\Pi_{1}(i) \triangleq \prod_{t=1}^{i} x_{1}(t), \Pi_{2}(i) \triangleq \prod_{t=1}^{i} x_{2}(t), \gamma_{1} \triangleq$ $\frac{b(1-b+\epsilon)}{(1-b)(b-\epsilon)}$ and $\gamma_{2} \triangleq \frac{b(1-b-\epsilon)}{(1-b)(b+\epsilon)}$. Similarly, the event of the market threshold portfolio $(b-\epsilon, b+\epsilon)$ hitting any of the thresholds first time at the $\tau$-th period, $\mathbf{b}^{\tau} \in \mathcal{E}_{\tau}^{\mathrm{fc}}$, can be defined as the intersections of the events [27]

$$
\begin{aligned}
& \mathbf{b}^{\tau} \in \mathcal{E}_{\tau}^{\mathrm{fc}} \equiv \bigcap_{i=1}^{\tau-1}\left\{\gamma_{2} \Pi_{1}(i) \leq \Pi_{2}(i) \leq \gamma_{1} \Pi_{1}(i)\right\} \\
& \bigcap\left[\left\{\Pi_{2}(\tau) \geq \gamma_{1} \Pi_{1}(\tau)\right\} \bigcup\left\{\Pi_{2}(\tau) \leq \gamma_{2} \Pi_{1}(\tau)\right\}\right],
\end{aligned}
$$

yielding the explicit definitions of the events $\mathbf{b}^{\tau} \in \mathcal{E}_{\tau}^{\mathrm{fc}}$ in (11) and $\mathbf{b}^{\tau} \in \mathcal{E}_{\tau}^{\text {nc }}$ in (10). The definitions of $\mathbf{b}^{\tau} \in \mathcal{E}_{\tau}^{\text {nc }}$ and $\mathbf{b}^{\tau} \in \mathcal{E}_{\tau}^{\text {fc }}$ can be readily extended for the case $m>2$ by using the updated definitions of $\Pi_{1}, \Pi_{2}, \ldots, \Pi_{m}$.

Using the quantitative definitions of the events $\mathbf{b}^{\tau} \in \mathcal{E}_{\tau}^{\mathrm{fc}}$ and $\mathbf{b}^{\tau} \in \mathcal{E}_{\tau}^{\text {nc }}$, we can express $P\left(\mathcal{E}_{\tau}^{\text {nc }}\right) T(\tau)$ as [27]

$$
\begin{aligned}
& P\left(\mathcal{E}_{\tau}^{\mathrm{nc}}\right) T(\tau)=\int_{0}^{\infty} \int_{\gamma_{2} \pi_{1}}^{\gamma_{1} \pi_{1}}\left(b \pi_{1}+(1-b) \pi_{2}\right) P\left(\Pi_{1}(\tau)=\pi_{1},\right. \\
& \left.\Pi_{2}(\tau)=\pi_{2}\right) P\left(\Sigma_{2}^{\tau} \in\left[\kappa-\theta_{1}, \kappa-\theta_{2}\right], \Sigma_{3}^{\tau} \in\left[\kappa-\theta_{1}, \kappa-\theta_{2}\right],\right. \\
& \left.\ldots, \Sigma_{\tau}^{\tau} \in\left[\kappa-\theta_{1}, \kappa-\theta_{2}\right]\right) \mathrm{d} \pi_{2} \mathrm{~d} \pi_{1},
\end{aligned}
$$


which follows from the definition of $\Sigma_{i}^{k}$ where $\kappa \triangleq \ln \frac{\pi_{2}}{\pi_{1}}$. The first probability in (12) can be calculated as [27]

$$
\begin{aligned}
& P\left(\Pi_{1}(\tau)=\pi_{1}, \Pi_{2}(\tau)=\pi_{2}\right)=\frac{1}{\pi_{1} \sqrt{2 \pi \tau \sigma_{1}^{2}}} e^{-\frac{\left(\ln \pi_{1}-\tau \mu_{1}\right)^{2}}{2 \tau \sigma_{1}^{2}}} \\
& +\frac{1}{\pi_{1} \sqrt{2 \pi \tau \sigma_{2}^{2}}} e^{-\frac{\left(\ln \pi_{2}-\tau \mu_{2}\right)^{2}}{2 \tau \sigma_{2}^{2}}}
\end{aligned}
$$

which follows since $\Pi_{1}(\tau) \triangleq \prod_{t=1}^{\tau} x_{1}(t)$ and $\Pi_{2}(\tau) \triangleq$ $\prod_{t=1}^{\tau} x_{2}(t)$ and we have $\Pi_{1}(\tau) \sim \ln \mathcal{N}\left(\tau \mu_{1}, \tau \sigma_{1}^{2}\right)$ and $\Pi_{2}(\tau) \sim \ln \mathcal{N}\left(\tau \mu_{2}, \tau \sigma_{2}^{2}\right)$.

Similarly we can express $P\left(\mathcal{E}_{\tau}^{\mathrm{fc}}\right) R(\tau)$ as [27]

$$
\begin{aligned}
& P\left(\mathcal{E}_{\tau}^{\mathrm{fc}}\right) R(\tau)=\int_{0}^{\infty} \int_{\gamma_{1} \pi_{1}}^{\infty}\left(\zeta_{1} \pi_{1}+\zeta_{2} \pi_{2}\right) P\left(\Pi_{1}(\tau)=\pi_{1}\right. \\
& \left.\Pi_{2}(\tau)=\pi_{2}\right) P\left(\Sigma_{2}^{\tau} \in\left[\kappa-\theta_{1}, \kappa-\theta_{2}\right], \Sigma_{3}^{\tau} \in\left[\kappa-\theta_{1}, \kappa-\theta_{2}\right]\right. \\
& \left., \ldots, \Sigma_{\tau}^{\tau} \in\left[\kappa-\theta_{1}, \kappa-\theta_{2}\right]\right) \mathrm{d} \pi_{2} \mathrm{~d} \pi_{1}+\int_{0}^{\infty} \int_{0}^{\gamma_{2} \pi_{1}}\left(\zeta_{3} \pi_{1}+\zeta_{4} \pi_{2}\right) \\
& P\left(\Pi_{1}(\tau)=\pi_{1}, \Pi_{2}(\tau)=\pi_{2}\right) P\left(\Sigma_{2}^{\tau} \in\left[\kappa-\theta_{1}, \kappa-\theta_{2}\right]\right. \\
& \left.\ldots, \Sigma_{\tau}^{\tau} \in\left[\kappa-\theta_{1}, \kappa-\theta_{2}\right]\right) \mathrm{d} \pi_{2} \mathrm{~d} \pi_{1}
\end{aligned}
$$

where the probability $P\left(\Pi_{1}(\tau)=\pi_{1}, \Pi_{2}(\tau)=\pi_{2}\right)$ can be obtained via (13).

Hence to calculate $P\left(\mathcal{E}_{\tau}^{\text {nc }}\right) T(\tau)$ and $P\left(\mathcal{E}_{\tau}^{\mathrm{fc}}\right) R(\tau)$, we need to calculate the probability $P\left(\Sigma_{2}^{\tau} \in\left[\kappa-\theta_{1}, \kappa-\right.\right.$ $\left.\left.\theta_{2}\right], \Sigma_{3}^{\tau} \in\left[\kappa-\theta_{1}, \kappa-\theta_{2}\right], \ldots, \Sigma_{\tau}^{\tau} \in\left[\kappa-\theta_{1}, \kappa-\theta_{2}\right]\right)$ in (12) and (14). We emphasize that the given multivariate probability cannot be calculated in a closed form [28], however there are some algorithms proposed in the literature to calculate it with small errors. In this paper, we use the randomized Quasi-Monte Carlo (QMC) algorithm, provided in [27,28].

\section{SIMULATIONS}

In this section, we illustrate the performance our algorithm under different scenarios. We use our algorithm over the historical data set collected from the New York Stock Exchange over a 22-year period $[9,14]$ and illustrate the average performance. In these simulations, we compare the performance of our algorithm with portfolio selection strategies from $[9,14$, 29].

To remove any bias on a particular stock pair, we show the average performance of the TRP algorithm over randomly selected stock pairs from the historical data set from [9]. The total set includes 34 different stocks, where the Iroquois stock is removed due to its peculiar behavior. We first randomly select pairs of stocks and invest using: the sequential TRP algorithm with the sequential ML estimators, the Cover's universal portfolio algorithm, the Iyengar's universal portfolio algorithm and the SCRP algorithm. The sequential selection of the optimal TRP parameters are performed similar to the previous case, i.e., we use ML estimators on an investment block of 1000 days and use the calculated optimal TRP in the next block of 1000 days. For each stock pair, we simulate the performance of the algorithms over 4651 days. In Fig. 1, we
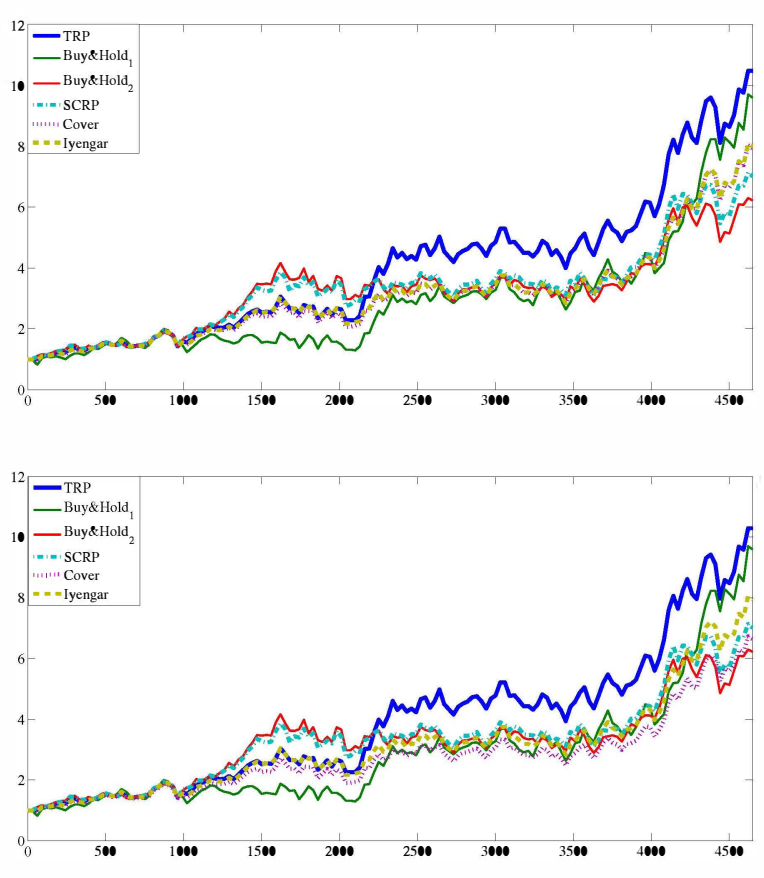

Fig. 1. Average performance of various portfolio investment algorithms on independent stock pairs under a moderate $(\mathrm{c}=0.01)$ and a hefty transaction cost $(\mathrm{c}=0.025)$.

present the wealth achieved by these algorithms, where the results are averaged over 10 independent trials. We present the achieved wealth over random sets of stock pairs under a hefty transaction cost $c=0.025$ and a moderate transaction cost $c=0.01$, where $c$ is the fraction paid in commission for each transaction, i.e., $c=0.01$ is a $1 \%$ commission, in Fig. 1 . We observe that the performance of the TRP algorithm readily outperforms the other algorithms for different transaction costs on this historical data set. Moreover, the relative gain is larger for the large transaction cost since the TRP approach, with the optimal parameters chosen as in this paper, can hedge more effectively against the transaction costs.

\section{CONCLUSION}

In this paper, we studied an important financial application, the portfolio selection problem, from a signal processing perspective. We investigated the portfolio selection problem in i.i.d. discrete-time markets having a finite number of assets, when the market levies proportional transaction fees for both buying and selling stocks. We introduced algorithms based on threshold rebalanced portfolios that achieve the maximal growth rate when the sequence of price relatives have the log-normal distribution from the well-known Black-Scholes model [24]. Under this setup, we provide an iterative relation that efficiently and recursively calculates the expected wealth in any i.i.d. market over any investment period. As predicted from our derivations, we significantly improve the achieved wealth over portfolio selection algorithms from the literature on the historical data set from [9]. 


\section{REFERENCES}

[1] IEEE Journal of Selected Topics in Signal Processing, "Special issue on signal processing methods in finance and electronic trading," .

[2] IEEE Signal Processing Magazine, "Special issue on signal processing for financial applications," .

[3] A. J. Bean and A. C. Singer, "Universal switching and side information portfolios under transaction costs using factor graphs," IEEE Journal of Selected Topics in Signal Processing, vol. 6, no. 4, pp. 351-365, August 2012.

[4] A. Bean and A. C. Singer, "Factor graphs for universal portfolios," in Proceedings of the Forty-Third Asilomar Conference on Signals, Systems and Computers, 2009, pp. 1375-1379.

[5] M. U. Torun, A. N. Akansu, and M. Avellaneda, "Portfolio risk in multiple frequencies," IEEE Signal Processing Magazine, vol. 28, no. 5, pp. 61-71, Sep 2011.

[6] A. Bean and A. C. Singer, "Universal switching and side information portfolios under transaction costs using factor graphs," in Proceedings of the ICASSP, 2010, pp. 1986-1989.

[7] A. Bean and A. C. Singer, "Portfolio selection via constrained stochastic gradients," in Proceedings of the SSP, 2011, pp. 37-40.

[8] M. U. Torun and A. N. Akansu, "On basic price model and volatility in multiple frequencies," in Proceedings of the SSP, June 2011, pp. 45-48.

[9] T. Cover, "Universal portfolios," Mathematical Finance, vol. 1, pp. 1-29, January 1991.

[10] T. Cover and E. Ordentlich, "Universal portfolios with side-information," IEEE Transactions on Information Theory, vol. 42, no. 2, pp. 348-363, 1996.

[11] D. P. Helmbold, R. E. Schapire, Y. Singer, and M. K. Warmuth, "Online portfolio selection using multiplicative updates," Mathematical Finance, vol. 8, pp. 325347, 1998.

[12] Y. Singer, "Swithcing portfolios," in Proc. of Conf. on Uncertainty in AI, 1998, pp. 1498-1519.

[13] V. Vovk and C. Watkins, "Universal portfolio selection," in Proceedings of the COLT, 1998, pp. 12-23.

[14] S. S. Kozat and A. C. Singer, "Universal semiconstant rebalanced portfolios," Mathematical Finance, vol. 21, no. 2, pp. 293-311, 2011.

[15] S. S. Kozat and A. C. Singer, "Switching strategies for sequential decision problems with multiplicative loss with application to portfolios," IEEE Transactions on Signal Processing, vol. 57, no. 6, pp. 2192-2208, 2009.
[16] S. S. Kozat and A. C. Singer, "Universal switching portfolios under transaction costs," in Proceedings of the ICASSP, 2008, pp. 5404-5407.

[17] S. S. Kozat, A. C. Singer, and A. J. Bean, "A treeweighting approach to sequential decision problems with multiplicative loss," Signal Processing, vol. 92, no. 4, pp. 890-905, 2011.

[18] T. M. Cover and C. A. Thomas, Elements of Information Theory, Wiley Series, 1991.

[19] M. H. A. Davis and A. R. Norman, "Portfolio selection with transaction costs," Mathematics of Operations Research, vol. 15, pp. 676713, 1990.

[20] M. Taksar, M. Klass, and D. Assaf, "A diffusion model for optimal portfolio selection in the presence of brokerage fees," Mathematics of Operations Research, vol. 13, pp. 277-294, 1988.

[21] A. J. Morton and S. R. Pliska, "Optimal portfolio manangement with transaction costs," Mathematical Finance, vol. 5, pp. 337-356, 1995.

[22] M. J. P. Magill and G. M. Constantinides, "Portfolio selection with transactions costs," Journal of Economic Theory, vol. 13, no. 2, pp. 245-263, 1976.

[23] G. Iyengar, "Discrete time growth
optimal investment http://www.ieor.columbia.edu/ gi10/Papers/stochastic.pdf.

[24] D. Luenberger, Investment Science, Oxford University Press, 1998.

[25] H. Markowitz, "Portfolio selection," Journal of Finance, vol. 7, no. 1, pp. 77-91, 1952.

[26] Z. Bodie, A. Kane, and A. Marcus, Investments, McGraw-Hill/Irwin, 2004.

[27] S. Tunc and S. S. Kozat, "Optimal investment under transaction costs: A threshold rebalanced portfolio approach," http://arxiv.org/pdf/1203.4156v1.pdf.

[28] A. Genz and F. Bretz, Computation of Multivariate Normal and $t$ Probabilities, Springer, 2009.

[29] G. Iyengar, "Universal investment in markets with transaction costs," Mathematical Finance, vol. 15, no. 2, pp. 359-371, 2005. 